\title{
Otimização de Redes de Comunicação de Dados como Suporte à Implantação de Smart Grids.
}

\author{
Daniel Victor Teixeira Lima ${ }^{1}$, Vanilze Vaz ${ }^{1}$, Francisco E. A. F. Júnior ${ }^{1}$ \\ Marcelino Silva da Silva ${ }^{1}$ \\ ${ }^{1}$ Instituto de Tecnologia - Universidade Federal do Pará (UFPA) \\ Caixa Postal 479 - 66.075-110 - Belém - PA - Brasil \\ \{danielufpa2013, vanilzevaz, jralbbuquerque\}@gmail.com, marcelino@ufpa.br
}

\begin{abstract}
The problem of optimal resource allocation consists in distribution of facilities to satisfy a criterion in a given scenario. The development of analytical models and/or computational simulations are necessary for the operational viability evaluation of a Smart Grid network's requirements. Thenceforth, an analytical model based on graph theory formalized the discussed location problem, which was solved through a genetic algorithm that takes into consideration the Smart Grids' quality of service requirements. The proposal is efficient as far as it finds an economically viable topology that met the technical requirements.
\end{abstract}

Resumo. O problema de alocação de recursos consiste na distribuição dos mesmos obedecendo a um determinado critério em dado cenário. A construção de modelos analíticos elou simulações computacionais se fazem necessárias para a avaliação da viabilidade operacional de um cenário com os requisitos de uma rede Smart Grid. Neste contexto, um modelo analítico baseado em grafos formalizou o problema de localização apresentado, o qual foi solucionado através de um algoritmo genético que leva em consideração os requisitos de qualidade de serviço para Smart Grids. A proposta é eficiente pois encontra uma topologia economicamente viável que atende os requisitos técnicos.

\section{Introdução}

Historicamente a eletricidade apresenta-se como uma das principais fontes de energia no mundo, impulsionando o desenvolvimento econômico e tecnológico das civilizações. Nos últimos anos, entretanto, notou-se um aumento da demanda energética em um contexto global [Meng et al. 2020], mobilizando pesquisadores a realizarem estudos cada vez mais complexos a fim de encontrar novas maneiras de gerenciar e produzir energia de uma forma mais otimizada e sustentável.

No cenário atual, a indústria e as organizações governamentais partilham da necessidade de elaborar uma solução que concilie o aumento da demanda energética, com a adoção de métodos renováveis de geração e distribuição de energia elétrica. Diante disso, surge uma nova forma de controlar e monitorar as redes elétricas, denominadas Smart Grids $(S G)$ ou Redes Elétricas Inteligentes, que agregam em sua definição a junção de práticas de sistemas de comunicação com sistemas elétricos de potência, com a proposta de uma rede elétrica estável, eficiente, segura e sustentável [Harwood 2018, Melo et al. 2018]. 
As SGs são constituídas de dispositivos que realizam trocas de dados entre si e unidades de controle dispersas pela rede elétrica, criando um fluxo de informação bidirecional que necessita atender um determinado padrão de QoS (Quality of Service), o qual varia de acordo com a aplicação analisada [Dileep 2020]. De acordo com [Yona et al. 2018], os padrões de QoS de uma SG diferenciam-se aos apresentados por uma rede comum de internet, com a análise dos requisitos de rede desde sua fase inicial de planejamento até a implementação, ponderando as melhores topologias e menores custos [Zhao et al. 2017].

O restante deste artigo está organizado da seguinte forma: na seção 2 é apresentada a relação de trabalhos futuros, bem como as contribuições desse artigo. Na seção 3 será abordada uma visão geral acerca da modelagem do problema de planejamento de redes. A seção 4 descreve a meta-heurística utilizada para resolver o problema de localização de p-medianas. Na seção 5 são apresentados os resultados obtidos, finalizando o trabalho na seção 6, a qual apresenta as considerações finais do estudo e sugestões para propostas futuras.

\section{Trabalhos Relacionados}

O cenário de Smart Grid apresenta estudos profundos e complexos utilizando técnicas computacionais, heurísticas e simulações a fim de desenvolver redes mais eficientes. Em [Endler et al. 2017], por exemplo, foi utilizado um AG (Algoritmo Genético) para otimizar a distribuição espacial de creches em Curitiba, comparando o desempenho da metaheurística em cenários de escalas distintas. De forma análoga, na abordagem proposta por [Qi et al. 2018], utilizou-se um AG para otimizar a construção de novas conexões e nós em um cenário WAMPAC (Wide Area Monitoring Protection and Control) para conectar diferentes redes PLC (Power Line Communication) levando em conta as restrições econômicas e de latência da rede.

Pesquisas sobre a viabilidade econômica da implantação de SGs também apresentam importante papel na literatura, como o de [Melo et al. 2018], no qual foi realizado um levantamento financeiro dos equipamentos, otimizando a alocação dos equipamentos conciliando o QoS aos impactos financeiros. Similarmente, [Alaqeel and Suryanarayanan 2019] desenvolveu uma framework de análise operacional que avalia os custos e benefícios da inserção de tecnologias SG à infraestrutura de uma rede elétrica. A ferramenta considera os benefícios econômicos, ambientais, robustez e de segurança denotando os ganhos monetários ao inserir tecnologias SG à rede.

Por sua vez, [Quiroga et al. 2019] propôs um modelo alternativo que descreve o processo de expansão da capacidade de transmissão e geração de energia elétrica, levando em consideração aspectos ambientais como a adoção de políticas locais que taxem as emissões de gases poluentes. Foram apresentadas projeções acerca do impacto da adoção dessas políticas em uma abordagem financeira e ambiental, permitindo a elaboração de estratégias tanto para investimentos a longo prazo quanto à tomada de decisões operacionais imediatas.

Dessa forma, nota-se que a maioria dos trabalhos correlatos a essa temática concentram-se na construção de novos modelos, levando em consideração principalmente fatores ambientais e análise do custo operacional da implantação de uma rede de comunicação de dados à uma aplicação SG. Observa-se que não há uma preocupação no uso de ferramentas de simulação para a análise dos resultados obtidos no modelo. 
Diante disso, as principais contribuições desse artigo estão contidas na apresentação de um novo modelo analítico baseado na teoria de grafos que avalia a viabilidade operacional da rede e o desenvolvimento de um AG para resolver os problemas de p-medianas em uma rede SG, validando o mesmo através do uso de simulações que abstraiam as características irrelevantes da rede otimizada, propondo assim uma análise da viabilidade operacional da mesma.

\section{Modelagem do Problema de Planejamento da Rede}

A construção de modelos mentais e simulações constituem a base do processo de pensamento do ser humano, a partir de tal mecanismo, é possível chegar à conclusões e realizar tomada de decisão considerando aspectos relevantes a um determinado problema [Choi and Kang 2013].

\subsection{Descrição e Tipificação do Problema}

O problema de alocação de recursos consiste em designar uma certa quantidade de tecnologias com o objetivo de encontrar suas localizações ótimas, constituindo, dessa forma, um problema de otimização de localização de p-medianas [Karatas et al. 2016]. Arquitetar a infraestrutura de uma rede de comunicação de dados para uma aplicação SG desempenha um importante papel na fase de planejamento e concepção da rede [Qi et al. 2018].

Definida a problemática relacionada ao modelo proposto, é necessária a elaboração de um método de planejamento sistemático e escalável para a concepção e organização da rede SG, tal processo é baseado em um questionamento inerente ao planejamento de qualquer rede de comunicação de dados "qual é a melhor topologia de comunicação de dados, considerando um padrão de QoS desejado?”.

Neste estudo foi implementado o modelo de comunicação de dados via domínio da distribuição de energia elétrica, optou-se por tal domínio porque o mesmo apresentase como o domínio mais complexo no que concerne à infraestrutura de comunicação de dados. O modelo possui caráter geral podendo ser aplicado nos demais domínios. A Figura 1 adaptada de [Sauter and Lobashov 2011] mostra o domínio de distribuição utilizado.

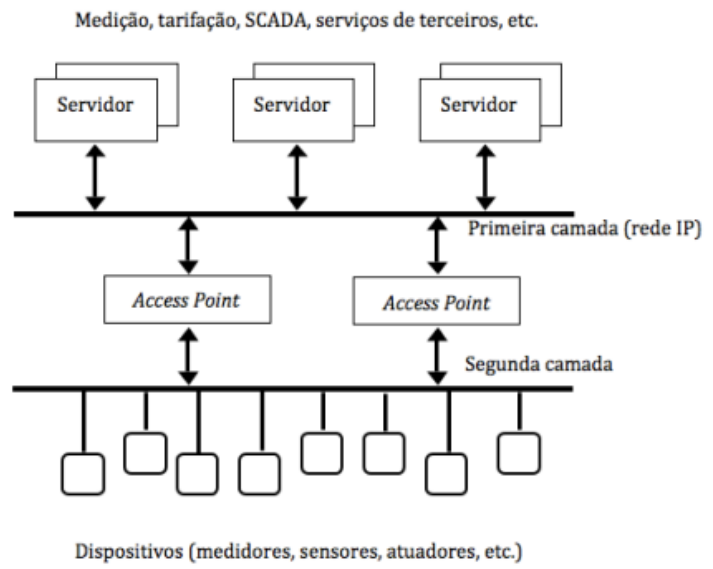

Figura 1. Arquitetura do sistema de comunicação de dados do domínio da distribuição. 
$\mathrm{Na}$ arquitetura da Figura 1 os servidores de aplicação estão conectados a primeira camada baseada em TCP/IP do modelo. A segunda camada do modelo corresponde as ligações dos equipamentos que realizam as trocas de dados entre os dispositivos e os servidores da área de cobertura das linhas de distribuição. A comunicação entre as camadas é realizada pelo AP (Access Point), que atua como mediador e Gateway da rede da segunda camada.

\subsection{Modelo Formal para Busca de Sub-grafos}

Dado um grafo não orientado e não-valorado $G(V, A)$, o qual abrange às especificações de todos os possíveis equipamentos de comunicação e ligações que conectam os elementos da rede, considerando-se o domínio da distribuição, o problema consiste em encontrar o sub-grafo não-valorado e não-direcionado $G^{\prime}\left(V^{\prime}, A^{\prime}\right)$, de maneira que o peso dos vértices e arestas seja ótimo quando $G^{\prime}\left(V^{\prime}, A^{\prime}\right)$ atender ao QoS da rede, minimizando o número de vértices dos grafos nos sentidos de upstream $\left(G^{\prime} u p\right)$ e downstream $\left(G^{\prime}\right.$ down).

Em um grafo $G(V, A)$ no qual $V$ é o conjunto de vértices e $A$ o conjunto de arestas de um grafo orientado e valorado $G_{u p}\left(V_{u p}, A_{u p}\right)$, no qual cada $v \in V$ forma um $V_{u p}$, atribui-se um peso e direção para $a \in A$ para formar $A_{u p}$, analogamente é formado o grafo orientado e valorado $G_{\text {down }}\left(V_{\text {down }}, A_{\text {down }}\right)$ a partir do qual serão formados o conjunto de vértices e arestas $V_{\text {down }}$ e $A_{\text {down }}$.

Assumindo um conjunto especial de vértices $W \subset V$ e $Z \subset V$, com $W \cap Z=$ $\emptyset$, deseja-se encontrar um sub-grafo $G^{\prime}\left(V^{\prime}, A^{\prime}\right)$, o qual pode formar $G_{u p}^{\prime}\left(V_{u p}^{\prime}, A_{u p}^{\prime}\right)$ e $G_{\text {down }}^{\prime}\left(V_{\text {down }}^{\prime}, A_{\text {down }}^{\prime}\right)$, de maneira a minimizar a ordem de $G^{\prime}$, sendo esse sujeito às restrições:

i. $V^{\prime}$ contém todos os vértices de $W$ e $Z, W \subset V^{\prime}$ e $Z \subset V^{\prime}$;

ii. Para cada par $w \in W$ e $z \in Z, T_{\text {nadj }}\left(G_{u p}^{\prime}, w, z\right) \leq R_{w, u p}$;

iii. Para cada par $w \in W$ e $z \in Z, T_{\text {nadj }}\left(G_{\text {down }}^{\prime}, z, w\right) \leq R_{w, \text { down }}$.

No qual $W$ corresponde ao conjunto de vértices que representam os Dispositivos Eletrônicos Inteligentes (DEIs), $Z$ é o conjunto de unitário que denota o AP e $R_{w}$ expressa a restrição de tempo definida pelos parâmetros de QoS das aplicações envolvendo $w$.

Nas situações onde a distância entre o servidor de aplicações e o AP é suficiente, de forma que gere um impacto significativo no atraso total do envio de dados entre dados do servidor e do AP $R_{w}$ irá denotar a parcela de atraso que a rede pode aplicar às transferências de dados das aplicações de $w$.

O sub-grafo obtido pelo modelo deve levar em considerações dois fatores: os requisitos de QoS da rede de comunicação de dados e a viabilidade econômica da topologia encontrada. Neste caso o modelo deve considerar à $R_{w}$ considere apenas aplicações indispensáveis ao sistema de distribuição elétrico.

\subsection{Formulação do Problema}

Considerando que os equipamentos de média e baixa tensão são os DEIs da segunda camada da arquitetura de referência e dado um grafo $G(V, A)$, que corresponde a um diagrama de conexões lógicas da $\mathrm{SG}$, no qual $V$ é o conjunto de vértices que representam os elementos da rede, e $A$ é o conjunto de arestas que corresponde à ligação direta entre dois elementos da rede, obtém-se um grafo que representa os nós fixos da rede $(\Delta, \nabla)$, repetidores $(R)$ e Gateways $(G)$, conforme ilustrado na Figura 2. 


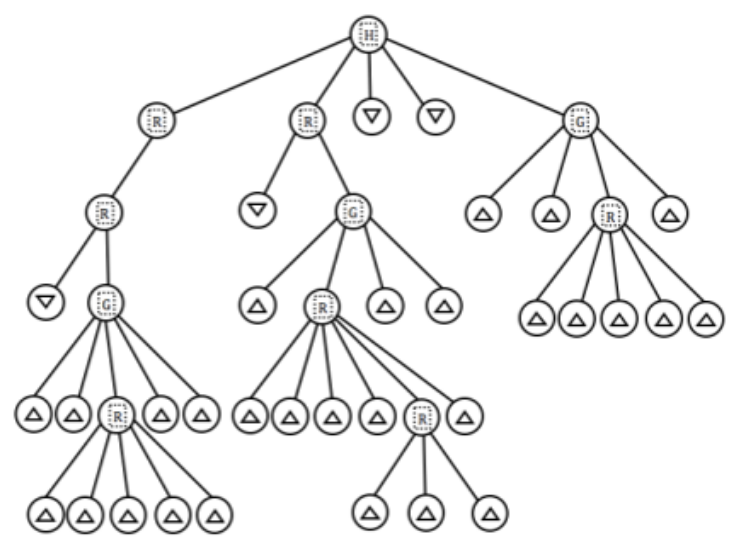

Figura 2. Grafo representando as conexões lógicas da rede PLC.

Sabendo que SGs possuem um fluxo bidirecional de informação, é necessário haver fluxo de dados nos sentidos de upstream e downstream entre o AP e os DEIs, gerando os grafos orientados $G_{\text {up }}\left(V_{\text {up }}, A_{\text {up }}\right)$ e $G_{\text {down }}\left(V_{\text {down }}, A_{\text {down }}\right)$.

Tendo em vista que o tráfego de dados entre os DEIS e os servidores da camada TCP/IP é representado pelo grafo valorado e orientado $G_{u p}\left(V_{u p}, A_{u p}\right)$, definiu-se $w_{u p}(a)$ como o peso das arestas $a \in A_{u p}$ que correspondem ao tempo de transmissão de um bit pelo canal $a$. A quantidade de dados transmitidos em bits entre os vértices $v \in V_{u p}$ é representada pelo peso $p_{u p}$ para os nós fixos da rede e uma somatória desses pesos para os nós intermediários.

O tempo total $T_{u p, a d j}$ pra que os dados provenientes do vértice $v_{i}$ sejam transmitidos para um vértice adjacente $v_{j}$, através de uma aresta $a=v_{i}, v j$ é apresentado na Equação 1 .

$$
T_{u p, a d j}\left(v_{i}, v_{j}\right)=p_{u p}\left(v_{i}\right) w_{u p}\left(a=\left\{v_{i}, v_{j}\right\}\right)
$$

$\mathrm{Na}$ Equação 1 é realizado o cálculo de atraso para o pior caso possível, pois ao considerar-se que $T_{u p, a d j}\left(v_{i}, v_{j}\right)$ é o atraso sofrido por um conjunto de dados, supõe-se que dentre todos os dados de $p_{u p}\left(v_{i}\right)$ os dados provenientes dessa aplicação serão os últimos a serem transmitidos.

O tempo total para a transmissão de dados $\left(T_{\text {up,nadj }}\right)$ de um determinado DEI até o AP é definido na Equação 2, a qual considera uma sequência de vértices $v$ e arestas $a$ que representam os DEIs e suas conexões, de maneira que remontem ao maior caminho de $v$ até o AP.

$$
\begin{array}{r}
T_{u p, n a d j}(v, A P)=T_{u p, a d j}(v, v 1)+T_{u p, a d j}(v 1, v 2)+T_{u p, a d j}(v 2, v 3)+\ldots \\
+ \\
+T_{u p, a d j}\left(v_{n}, A P\right)+n t_{0}
\end{array}
$$

O termo $t_{0}$ da Equação 2 representa o tempo para que cada um dos $n$ nós retransmitam os dados recebidos, levando em conta o tempo de processamento do sinal, o valor adotado para tal processo é de $0,07 \mathrm{~ms}$, valor esse aferido em medições feitas no equipa- 
mento. O cálculo do atraso no sentido downstream obedece às mesmas formulações apresentadas pelo sentido upstream, mudando somente o sentido da transmissão. A Equação 3 , descreve que a partir do cálculo da capacidade de transmissão $\left(c_{a}\right)$ dos vértices e arestas do grafo que representa a rede é possível estimar o tempo de transmissão de um bit $(w)$.

$$
\omega(a)=\frac{1}{c_{a}}
$$

Dado que o número de pontos possíveis para instalar os equipamentos é finito, é viável montar um grafo que inclua todos os equipamentos da rede de comunicação de dados. A partir desse grafo, é possível encontrar um sub-grafo que minimize o número de DEIs utilizados e que atenda aos requisitos necessários para a implementação das aplicações SG.

\subsection{Estudo de Caso}

Esse artigo utiliza como cenário o modelo de referência do IEEE para teste de distribuição com 13 nós [Kersting 2001]. Considerou-se que cada nó da Figura 3 representa um DEI, o qual é utilizado por aplicações de proteção e análise da qualidade de energia.

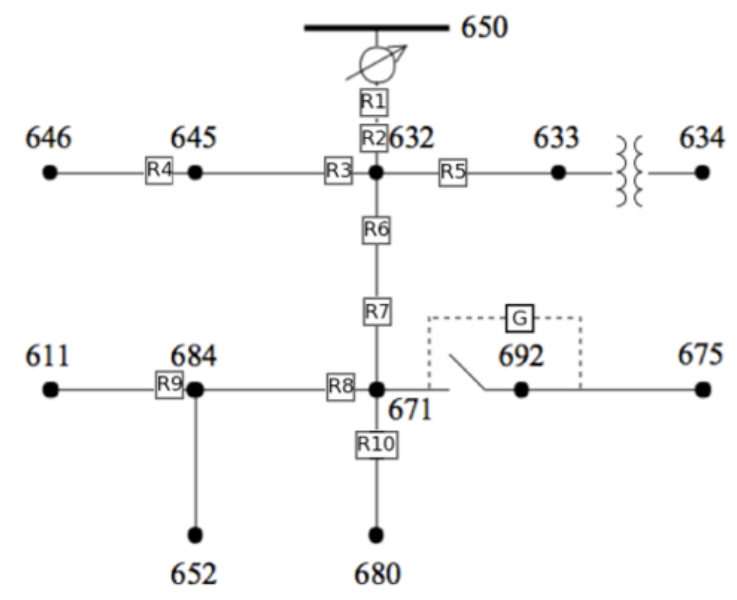

Figura 3. Possíveis Localizações dos equipamentos PLC.

Sabendo que a localização dos DEIs e do AP é fixa e definida de acordo com as necessidades do sistema elétrico, o problema consiste em otimizar a alocação dos nós intermediários da rede de comunicação de dados, para que sejam estabelecidas conexões com o AP, como pode ser observado na Figura 3. Os números atribuídos aos nós servem como identificadores para cada nó, os símbolos presentes no modelo IEEE utilizado são: fonte de corrente, transformador e um interruptor simples, presentes em 650, entre os nós 633 e 634 e entre os nós 671 e 692, respectivamente.

A partir das considerações realizadas, foram montadas as matrizes de adjacência para upstream e downstream, contendo todos os vértices e arestas possíveis de $G(V, A)$. As taxas de transmissão de dados das conexões foram analiticamente calculadas baseando-se em [Thayoob et al. 2010], a partir do qual foram definidos os pesos das arestas, que correspondem ao tempo de transmissão de um bit. 


\subsection{Parâmetros do Modelo}

A tecnologia escolhida para modelar o cenário descrito na Figura 3 da rede de comunicação de dados foi a Power Line Communication (PLC), devido ao fato das linhas de distribuição de energia elétrica serem o canal de transmissão de dados e do alto grau de capilaridade e extensão das linhas energéticas se comparada à outras tecnologias [Galli et al. 2011].

A quantidade de dados transmitidos, a relevância dos dados e o atraso máximo aceitável são determinados a partir do tipo de aplicação na qual a rede de dados se enquadra. A norma IEC 61850 [Adamiak et al. 2009] foi adotada para tal, pois esta apresenta uma classificação dos tipos de mensagens transmitidas em uma SG. As mensagens podem ser divididas em três tipos principais, detalhados na Tabela 1.

Tabela 1. Detalhes de cada tipo de mensagem

\begin{tabular}{|c|c|c|c|}
\hline Classe da Mensagem & Payload & Atraso Máximo & Importância \\
\hline Tipo 3 & 1024 bits & $100 \mathrm{~ms}$ & Baixa \\
\hline Tipo 2 & 128 bits & $50 \mathrm{~ms}$ & Média \\
\hline Tipo 1 & 1 bit & $1 \mathrm{~ms}$ & Alta \\
\hline
\end{tabular}

A norma IEC especifica o payload a ser transmitido, mas não leva em consideração os dados gerados pelo overhead do pacote, deixando a cargo do projetista essa tarefa. Nesse artigo, para os pacotes utilizados na simulação, o overhead gerado pelos protocolos Real Time Protocol (RTP), User Datagram Protocol (UDP) e Internet Protocol (IP) é de 40 bytes para cada tipo de mensagem.

\section{Meta-heurística Utilizada}

Para encontrar o melhor subconjunto de nós que atendam aos requisitos das aplicações SG, foi desenvolvido um AG utilizando o software de modelagem matemática MATLAB ${ }^{1}$.

A codificação dos cromossomos constituintes da população submetida ao AG proposto se dá com definição de que cada membro da população representa uma possível solução para o problema de localização de p-medianas, logo cada cromossomo simboliza uma topologia da rede de comunicação PLC candidata à solução ótima.

A rede de comunicação PLC possui um Gateway e dez repetidores como nós intermediários e os 13 DEIs fixos do modelo de teste IEEE. Considerando um cromossomo $c$ e que o mesmo simboliza uma topologia de rede, $c$ é representado por uma matriz $m$, na qual cada linha e coluna de $m$ corresponde a uma conexão lógica entre os DEIs constituintes da rede. A codificação de $c$ é mostrada na Tabela 2.

Tabela 2. Codificação dos cromossomos da população

\begin{tabular}{|c|c|c|c|c|c|c|c|c|c|c|c|c|}
\hline DEIs & 632 & 645 & 646 & 633 & 634 & 671 & 684 & 611 & 652 & 680 & 692 & 675 \\
\hline EQ & AP & R1 & R2 & R3 & R4 & R5 & R6 & R7 & R8 & R9 & R10 & G \\
\hline
\end{tabular}

A primeira linha da Tabela 2 corresponde aos DEIs do modelo de referência. Foram adicionados os nós intermediários da rede enumerados de 1 a 12 aos DEIs, com

\footnotetext{
${ }^{1}$ Disponível em: https://www.mathworks.com/ . Acessado em 06 de Mar de 2020.
} 
1 representando uma conexão do DEI correspondente ao AP, os números de 2 a 11 ilustrando o conjunto de repetidores e 12 simbolizando a conexão com o Gateway. A segunda linha do cromossomo condiz às conexões realizadas pelos nós intermediários apresentados anteriormente, para tal subtrai-se 1 do valor apresentado na primeira linha, a partir daí a coluna correspondente de tal equipamento é selecionada e o processo é repetido até que se tenha uma conexão direta do nó intermediário com o AP.

A seleção por torneio foi o método utilizado para eleger os indivíduos aptos à fase de cruzamento, caracterizada pela realização da troca de genes entre os indivíduos. Nesse artigo foi adotado o cruzamento de corte em um ponto aleatório para cada linha constituinte do cromossomo apresentado na Tabela 2. As escolhas de tais métricas justificam-se pelo fato de a seleção por torneio ter pressão seletiva reduzida, o cruzamento de corte de um ponto foi escolhido porque o mesmo é capaz de gerar novos indivíduos e ao mesmo tempo manter a estrutura do grafo usado para modelar o problema.

A mutação do AG proposto deu-se de forma que, dada que a probabilidade de mutação fosse atingida, um gene do cromossomo sofre uma alteração dentro das restrições pertinentes à topologia de rede gerada pelo modelo. Elitismo simples foi utilizado para garantir que o indivíduo mais apto seja permeado às próximas gerações do AG.

A aptidão de cada cromossomo $c, A_{c}$, é descrita na Equação 4.

$$
\begin{aligned}
A_{c}=\frac{N_{P L C}-N_{P L C U}}{N_{P L C}}+ & \sum_{N_{D E I}}^{i=1} \min \left(\frac{R_{u p, i}}{T_{u p, n a d j}(i, A P)}, 1\right)+\ldots \\
& +\sum_{N_{D E I}}^{i=1} \min \left(\frac{R_{\text {down }, i}}{T_{\text {down }, \text { nadj }}(A P, i)}, 1\right)
\end{aligned}
$$

Onde NPLC é o número total de equipamentos PLC possíveis de serem utilizados, $N_{D E I}$ é o número de DEIs, $R_{u p, i}$ é o atraso máximo aceitável definido para o DEI no sentido de upstream e $R_{\text {down }, i}$ é o atraso máximo para os DEIs no sentido de downstream. Levando em conta a topologia do cromossomo $c, N P L C U$ é o número de equipamentos PLC utilizados, $T_{\text {up,nadj }}(i, A P)$ é o atraso calculado para o envio de mensagens do DEI $i$ ao AP no sentido de upstream e $T_{\text {down,nadj }}(A P, i)$ é o mesmo atraso, porém para o sentido de downstream.

$\mathrm{Na}$ Equação 4 o primeiro termo da mesma denota o ganho em relação ao número de equipamentos PLC utilizados. O segundo e o terceiro termo da equação ilustram os ganhos relacionados ao cumprimento das restrições de upstream e downstream, respectivamente, tendo em vista que quanto maior $T_{u p, n a d j}(i, A P)$ e $T_{\text {down,nadj }}(A P, i)$ em relação a $R_{u p, i}$ e $R_{\text {down }, i}$ pior será o desempenho do sistema.

\section{Resultados}

O modelo foi submetido a 30 execuções do AG, onde foram retiradas as médias dos atrasos de cada execução, foi adotada uma população de 50 indivíduos com taxa de mutação de 0,03 , usando o método de torneio para o cruzamento dos cromossomos e com elitismo simples, os parâmetros adotados no AG são de natureza empírica. 
A topologia do cromossomo apresentada na Figura indica que os DEIs R1, R8, R6, R7 e G foram utilizados como nós intermediários da rede. Os valores da segunda linha de $c$ excluídos dos resultados se deve ao fato de deles não se conectarem a nenhum nó fixo da rede da primeira linha. A topologia do cromossomo encontrada pelo AG é apresentada na Figura 4, que mostra o arranjo formado pelos roteadores (preto), DEIs (azul) e pelo AP (verde). Os resultados de atraso máximo da topologia são mostrados na Tabela 3.

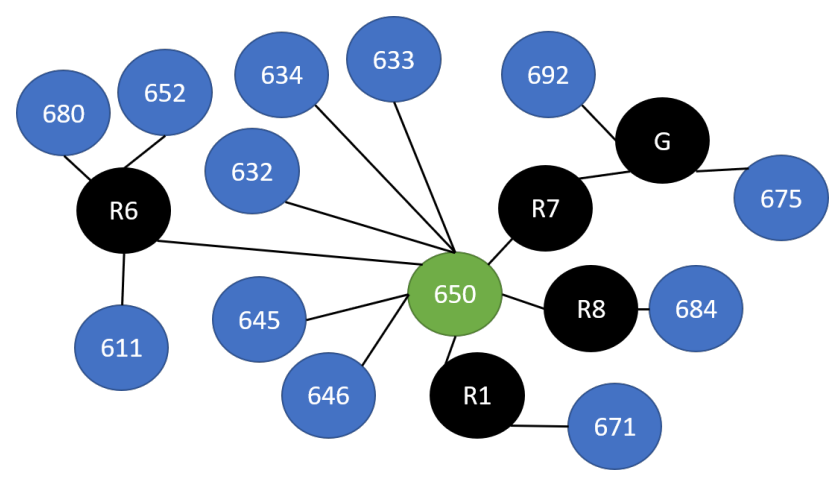

Figura 4. Topologia lógica encontrada pelo AG.

Tabela 3. Resultados de atraso acumulado para a topologia da Figura 4

\begin{tabular}{|c|l|c|}
\hline DEI & Atraso (ms) & Restrição atendida \\
\hline $632,645,633,646,634$ & 0,0742 & $\operatorname{sim}$ \\
\hline 671 & 0,0992 & $\operatorname{sim}$ \\
\hline 684 & 0,1982 & $\operatorname{sim}$ \\
\hline 611 & 0,1473 & $\operatorname{sim}$ \\
\hline 652 & 0,1473 & $\operatorname{sim}$ \\
\hline 680 & 0,1473 & $\operatorname{sim}$ \\
\hline 692 & 0,2252 & $\operatorname{sim}$ \\
\hline 675 & 0,1473 & $\operatorname{sim}$ \\
\hline
\end{tabular}

A partir dos resultados da topologia, verifica-se que os DEIs 632, 645, 646, $633 \mathrm{e}$ 634 estão ligados diretamente ao AP, visto que existem rotas alternativas disponíveis no sistema que mostram-se inviáveis pelo aumento expressivo do número de equipamentos utilizados ou no sobrecarregamento de um equipamento, com todos os nós atendendo ao QoS.

A média do atraso dos melhores indivíduos (AGB) encontrados pelo AG, comparada ao atraso apresentado pela média das topologias geradas aleatoriamente na primeira execução do algoritmo (AG1), é mostrada na Figura 5. 


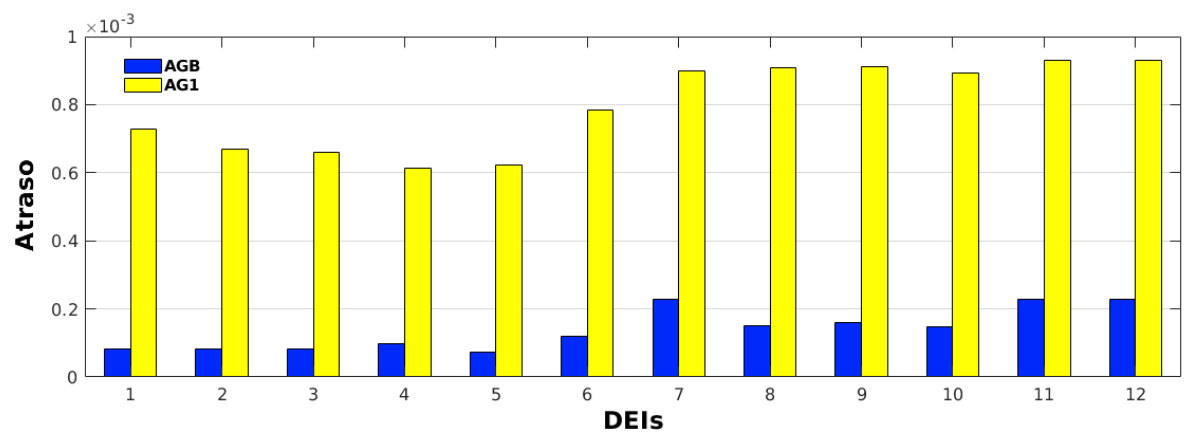

Figura 5. Atraso médio da primeira geração do AG em relação à média dos melhores indivíduos.

As simulações foram desenvolvidas utilizando o software NS-32 , e foram baseadas no modelo descrito nesse artigo, além de utilizar os 3 tipos de mensagens contidos na Tabela 1 com um overhead de 40 bytes gerado pela pilha de protocolos, os pacotes foram transmitidos do nó 675 em direção ao nó 650, o qual corresponde ao ponto de acesso da rede. O critério utilizado para a escolha do nó 675 se dá, principalmente, pelo número de nós intermediários entre o ponto de acesso e o dispositivo em questão.

A Tabela 4 mostra os resultados da simulação para o envio das mensagens de tipo 1,2 e 3 com seus respectivos atrasos encontrados pela simulação do envio de pacotes.

Tabela 4. Resultados da Simulação do melhor indivíduo do AG

\begin{tabular}{|c|c|c|c|}
\hline Classe da Mensagem & Payload & Atraso Calculado (ms) & Restrição Atendida \\
\hline Tipo 3 & 1344 & 2,3473 & SIM \\
\hline Tipo 2 & 448 & 0,9137 & SIM \\
\hline Tipo 1 & 321 & 0,7105 & SIM \\
\hline
\end{tabular}

Como pode-se observar os requisitos da rede foram atendidos para todos os tipos de mensagens que são enviadas pela rede. Porém vale ressaltar que o intuito da simulação computacional é de apenas validar o modelo em uma primeira instância, a validação completa do modelo só pode ser feita através da simulação da rede proposta com os equipamentos e canais em ambiente PLC.

A Tabela 5 mostra os resultados da simulação para o indivíduo da população inicial com melhor aptidão, $c=\left[\begin{array}{l}3 \\ 768\end{array} 61911811812\right.$ 12; 135371117111178 , o qual indica que os equipamentos R2, R3, R5, R6, R7, R8, R10 e G são utilizados na construção da rede simulada.

Tabela 5. Resultados da Simulação de um indivíduo da primeira geração do AG.

\begin{tabular}{|c|c|c|c|}
\hline Classe da Mensagem & Payload & Atraso Calculado (ms) & Restrição Atendida \\
\hline Tipo 3 & 1344 & 0,7546 & SIM \\
\hline Tipo 2 & 448 & 0,5395 & SIM \\
\hline Tipo 1 & 321 & 0,5091 & SIM \\
\hline
\end{tabular}

\footnotetext{
${ }^{2}$ Disponível em: https://www.nsnam.org/about/. Acessado em: 10 de Mar de 2020
} 
Os dados mostrados na Tabela 5 indicam que os requisitos da rede atenderam às especificações de QoS. Ao comparar os dados contidos nas Tabelas 4 e 5 observa-se que os atrasos da topologia simulada na Tabela 5 possuem atrasos menores para todos os tipos de mensagem, porém a rede encontrada na primeira geração faz o uso de 8 equipamentos PLC, enquanto que a rede encontrada pelo AG consegue atender a todos os requisitos com apenas 5 dispositivos PLC, tornando-se assim uma alternativa com melhor relação custo benefício.

\section{Conclusão}

Com base nos resultados obtidos na execução dos experimentos do AG e os resultados apresentados pelas simulações, para o problema em questão, são necessários 4 repetidores e um Gateway para atender os requisitos de QoS das aplicações exigidas pela rede PLC. Observa-se também que os atrasos calculados pela topologia encontrada pelo algoritmo estão sempre inferiores às exigências da rede. Dessa forma, pode-se inferir que as mensagens da aplicação prioritárias sejam entregues dentro do intervalo máximo aceitável, mesmo em um cenário onde as mesmas sejam entregues em última instância.

A partir da análise dos gráficos da média da população e das métricas de QoS avaliadas, notou-se a evolução das soluções em direção a um ponto ótimo em dois momentos: No primeiro momento as soluções convergiram rapidamente em direção a uma solução sub-ótima, no segundo momento houve uma evolução mais gradativa das soluções geradas pelos algoritmos.

A simulação do modelo proposto mostra que é possível validar o mesmo, uma vez que os valores de atraso encontrados após a submissão do modelo à simulação de tráfego de pacotes indicam que a topologia obedece a todos os requisitos de QoS da rede.

Como trabalhos futuros, propõe-se a simulação do cenário descrito neste trabalho com outras tecnologias de transmissão, a melhora dos níveis de abstração da simulação, com a representação de diferentes canais e tecnologias, o emprego de outras técnicas de Inteligência Computacional para a solução do modelo, além da adaptação do modelo de maneira que o mesmo leve em conta fatores como taxas de emissões de gases poluentes.

\section{Referências}

Adamiak, M., Baigent, D., and Mackiewicz, R. (2009). Iec 61850 communication networks and systems in substations: An overview for users. The Protection \& Control Journal, pages 61-68.

Alaqeel, T. A. and Suryanarayanan, S. (2019). A comprehensive cost-benefit analysis of the penetration of Smart Grid technologies in the Saudi Arabian electricity infrastructure. Utilities Policy, 60(June):100933.

Choi, B. K. and Kang, D. (2013). Modeling and Simulation of Discrete-Event Systems. John Wiley \& Sons.

Dileep, G. (2020). A survey on smart grid technologies and applications. Renewable Energy, 146:2589-2625.

Endler, K. D., Scarpin, C. T., and Steiner, M. T. A. (2017). Algoritmo Genético Para Resolução Do Problema Da Localização De Centros Públicos De Educação Infantil. Xlix Sbpo. 
Galli, S., Scaglione, A., and Wang, Z. (2011). For the grid and through the grid: The role of power line communications in the smart grid. Proceedings of the IEEE, 99(6):9981027.

Harwood, P. (2018). Clustering-driven equipment deployment planner and analyzer for wireless non-mobile networks applied to smart grid scenarios. Master's thesis, Federal University of Pará, Belém - PA. An optional note.

Karatas, M., Razi, N., and Tozan, H. (2016). A comparison of p-median and maximal coverage location models with Q-coverage requirement. Procedia Engineering, 149(June):169-176.

Kersting, W. H. (2001). Radial distribution test feeders. Proceedings of the IEEE Power Engineering Society Transmission and Distribution Conference, 2(WINTER MEETING):908-912.

Melo, P. T. d. L. et al. (2018). Total cost of ownership for communications infrastructure of smart grid. Proceeding of ACM Conference, 1:1-7.

Meng, J., Hu, X., Chen, P., Coffman, D. M., and Han, M. (2020). The unequal contribution to global energy consumption along the supply chain. Journal of Environmental Management, 268(February):110701.

Qi, F., Yu, P., Chen, B., Li, W., Zhang, Q., Jin, D., Zhang, G., and Wang, Y. (2018). Optimal Planning of Smart Grid Communication Network for Interregional Wide-Area Monitoring Protection and Control System. 2018 IEEE International Conference on Energy Internet (ICEI), pages 190-195.

Quiroga, D., Sauma, E., and Pozo, D. (2019). Power system expansion planning under global and local emission mitigation policies. Applied Energy, 239(March 2018):1250-1264.

Sauter, T. and Lobashov, M. (2011). End-to-end communication architecture for smart grids. IEEE Transactions on Industrial Electronics, 58(4):1218-1228.

Thayoob, Y. H. M., Ariffin, A. M., and Sulaiman, S. (2010). Analysis of high frequency wave propagation characteristics in medium voltage XLPE cable model. ICCAIE 2010 - 2010 International Conference on Computer Applications and Industrial Electronics, (Iccaie):665-670.

Yona, L., Natalia, C. F., et al. (2018). Smart grid communication : Requirements and scada protocols analysis. Simpósio Brasileiro de Sistemas Elétricos (SBSE), 1:1-6.

Zhao, P., Chen, X., Yu, P., Li, W., Qiu, X., and Guo, S. (2017). Risk assessment and optimization for key services in smart grid communication network. Proceedings of the IM 2017 - 2017 IFIP/IEEE International Symposium on Integrated Network and Service Management, pages 600-603. 